\title{
Estudo da dinâmica das motocicletas em frenagens e curvas: $O$ efeito da técnica do piloto e da condição da estrada
}

Motorcycle dynamics in braking and curves: The effect of the rider technique and road condition

\author{
Fábio Santana Magnani ${ }^{1}$, Saulo dos Santos Cunha*1 \\ ${ }^{1}$ Universidade Federal de Pernambuco, Recife, PE, Brasil
}

Recebido em 2 de Outubro de 2016. Revisado em 25 de Novembro de 2016. Aceito em 27 de Novembro de 2016

\begin{abstract}
O estudo das motocicletas apresenta um interessante exemplo de ensino que combina teoria, tecnologia e prática. Neste artigo, apresentamos um estudo dinâmico típico efetuado na disciplina Engenharia da Motocicleta, UFPE. O problema em questão é determinar quando uma motocicleta deve iniciar a sua frenagem e qual é a máxima velocidade em que pode fazer uma determinada curva. Para estudar esse movimento, modelamos a dinâmica da motocicleta em linha reta e depois em curva, considerando as forças inerciais, as forças resistivas, e as forças controladas pelo piloto (propulsão e frenagem). Os modelos matemáticos são então usados para estudar a mínima distância para iniciar a frenagem e a máxima velocidade possível na curva, considerando limites de aderência e a possibilidade de perda de contato de uma das rodas. Como resultado, concluímos que, do ponto de vista da segurança, é importante a consideração das forças resistivas no modelo da velocidade máxima em curva, mas não no cálculo da distância mínima de frenagem. Outro resultado importante é que a técnica ótima de frenagem (proporção do freio dianteiro e traseiro) é função do coeficiente de atrito, não sendo interessante recomendar aos pilotos uma proporção universal.
\end{abstract}

Palavras-chave: motocicleta, frenagem, dinâmica na curva.

The study of motorcycles presents an interesting example of teaching that combines theory, technology and application. In this paper, we present a typical dynamic study conducted in the discipline of Motorcycle Engineering, UFPE. The problem is to determine when a motorcycle should begin its breaking and what would be the maximum speed to make a curve. To study this phenomenon, we model the dynamics of the motorcycle on straight and curved paths, considering the inertial forces, resistive forces and the forces controlled by the rider (propulsion and braking). The models are then used to study the minimum distance to start the braking and the maximum possible cornering speed, considering the limits of adhesion and the possibility of loss of contact of one of the wheels. As result, we conclude, from a safety point of view, that it is important to consider the resistive forces on the model of maximum cornering speed, but not in the calculation of the minimum braking distance. Another important result is that the optimum braking technique (ratio of front and rear braking) is a function of the friction coefficient, so it is not interesting to recommend to the riders a universal proportion.

Keywords: motorcycle, braking, curve dynamics.

\section{Introdução}

Desde 2010, a disciplina Engenharia da Motocicleta vem sendo oferecida pelo Curso de Graduação em Engenharia Mecânica da UFPE. A disciplina começa estudando os problemas gerais ligados às motoci-

*Endereço de correspondência: saulodsc2@hotmail.com cletas, como acidentes, poluição, preconceito e monopólio, e, logo depois, descreve a tecnologia do veículo, incluindo motor, quadro, direção, pneus, etc. Concluídos esses $20 \%$ da disciplina, começa a parte principal, dedicada à modelagem matemática da motocicleta: propulsão, frenagem, manobras e amortecimento. Essa combinação é bastante rica do 
ponto de vista pedagógico, pois revisa os conceitos teóricos da dinâmica que os estudantes aprenderam em disciplinas preliminares, inclui as limitações tecnológicas encontradas na prática, e, principalmente, mostra como uma modelagem relativamente simples pode ser usada para auxiliar na solução de problemas práticos. Do ponto de vista didático, um ponto interessante é que a motocicleta é um veículo bastante atraente entre os jovens, o que ajuda muito na motivação durante as aulas, trabalhos e avaliações.

Neste artigo, iremos mostrar como um modelo simples, basicamente de balanço de forças e de momentos, pode explicar o comportamento das motocicletas em frenagens e curvas. Adicionalmente, esse modelo pode ainda proporcionar respostas práticas, como a melhor técnica para o piloto usar durante a frenagem, a distância mínima para a redução de velocidade e a máxima velocidade na qual uma motocicleta consegue fazer uma determinada curva. Mas, antes de começar a apresentação do modelo dinâmico, iremos contextualizar o problema geral.

O número de acidentes com motocicletas no Brasil, bem como as suas consequências, são bem conhecidos. No entanto, não existem muitos estudos técnicos sobre as reais causas desses acidentes. A grande maioria dos trabalhos é feita nos leitos dos hospitais ou então baseados nos registros dos acidentes, não permitindo, portanto, nada mais que conjecturas sobre as reais causas dos acidentes. No mundo todo, os dois únicos trabalhos completos sobre as causas dos acidentes são de Hurt et al. [1] e ACEM [2]. Nesses trabalhos, equipes formadas por médicos e engenheiros se deslocavam aos locais dos acidentes para analisar quantitativamente o que realmente acontecera. Os resultados eram então comparados com situações de controle em vias próximas.

Em 2010, a Organização Mundial da Saúde foi convidada por alguns países a preparar um relatório com a avaliação do estado de segurança rodoviária global e para monitorar o progresso ao longo da década [3]. Essa ação indicou claramente a preocupação dos governantes ao redor do globo com os efeitos devastadores dos acidentes de trânsito.

No Brasil, na década de 1990, políticas públicas do governo federal incentivaram a fabricação, a compra e o uso das motocicletas. A frota desses veículos aumentou de 1,5 milhão em 1990 para 17 milhões no final de 2012. Por causa da sua versatilidade, baixo custo e conveniência pessoal, as motos passaram a ser usadas intensamente no transporte de pequenas mercadorias e pessoas, para o trabalho e para a escola, em áreas urbanas e rurais. Infelizmente, o aumento no número de motocicletas foi acompanhado pelo aumento dos acidentes com esses veículos [4].

As causas dos acidentes são variadas: configuração dos veículos, imprudência e imperícia dos condutores (tanto das motocicletas quanto dos automóveis), além do próprio projeto e das condições das vias. Em relação ao projeto das vias, na concepção e na implantação da segurança no trânsito, a sinalização é um fator básico que influencia a percepção dos usuários para as condições da via. De acordo com o CONTRAN [5], a sinalização de regulamentação tem por finalidade transmitir aos usuários as condições, proibições, obrigações ou restrições no uso das vias urbanas e rurais. Assim, o desrespeito aos sinais de regulamentação constitui infrações previstas Código de Trânsito Brasileiro [6].

Como boa prática de segurança, a redução da velocidade regulamentada para um trecho, em relação ao trecho imediatamente anterior, deve ser feita com base em estudos de engenharia que levem em conta diversos fatores, tais como o tempo de reação do condutor e a distância de frenagem em função da redução desejada. Por ser universal, a placa de sinalização deve levar em conta os diversos veículos que circulam pelas vias, como automóveis, motocicletas e caminhões.

No caso das motocicletas, há um complicador nessa análise, pois os freios traseiro e dianteiro são acionados de forma independente. Fabricantes [7] e acadêmicos [8] defendem que o domínio de uma boa técnica de frenagem (i.e., escolher a proporção correta entre a aplicação do freio dianteiro e do traseiro) permite reduzir em mais de $50 \%$ a distância de parada. Inversamente, conclui-se que o uso de uma má técnica pode então aumentar muito a distância de frenagem, podendo ocasionar queda ou colisão.

A capacidade da frenagem das motocicletas depende diretamente da aderência entre o pneu e o pavimento da pista, e, desta forma, deve-se evitar a todo custo que a utilização da aderência seja reduzida. Algumas das situações que provocam a redução da aderência são o bloqueio das rodas durante as frenagens e o deslizamento durante a aceleração, casos em que o pneu, ao invés de rolar, passa a escorregar sobre o pavimento da pista.

Assim, explicar o comportamento de uma motocicleta durante a frenagem não é trivial, pois envolve 
tanto a modelagem da dinâmica do veículo quanto das escolhas que o piloto realiza.

As referências clássicas para o estudo da dinâmica de veículos de duas rodas são Cossalter [8] no caso das motocicletas e Wilson [9] para as bicicletas. O presente trabalho é baseado nesses autores clássicos, mas com três contribuições. Em primeiro lugar, a inclusão das forças resistivas nos modelos de frenagem e de curva. Segundo, o acoplamento das duas manobras (frenagem e curva) para o estudo do problema específico de segurança. Terceiro, a consideração integrada de fenômenos indesejáveis, como a perda de aderência e o stoppie (perda de contato da roda traseira com o piso durante a frenagem).

Na Seção 2 apresentamos a modelagem matemática dos fenômenos. Depois, na Seção 3, esses modelos são usados para determinar a distância mínima de frenagem e a máxima velocidade da motocicleta em curva. Na Seção 4 discutimos em que casos é importante a consideração das forças resistivas e concluímos que as recomendações para colocação de placas sinalizadoras frenagem são seguras.

\section{Materiais e métodos}

Nesta seção, primeiramente são apresentadas as equações básicas da dinâmica das motocicletas e, em seguida, como essas equações são arranjadas especificamente para o presente estudo sobre frenagens e curvas.

\subsection{Fundamentos da dinâmica da motocicleta na reta}

Durante o movimento, a motocicleta sofre a ação de variadas forças, como apresentadas na Fig. 1. Algumas dessas forças provocam o movimento para frente, enquanto outras tendem a resistir ao movimento.

A força aplicada na roda traseira é apresentada pela equação (1). O primeiro termo do lado direito é a reação do piso à força vinda do motor, a qual depende da potência máxima do motor em uma determinada rotação $(\mathrm{P})$, da velocidade da motocicleta $(\mathrm{V})$ e do coeficiente de abertura do acelerador $(\alpha)$. O segundo termo é a força de frenagem, que depende do coeficiente de utilização do freio $\left(\mu_{T}\right)$ e da força normal $\left(N_{T}\right)$. Dependendo da atuação do piloto no acelerador $(\alpha)$ ou no freio $\left(\mu_{T}\right)$, ambos os

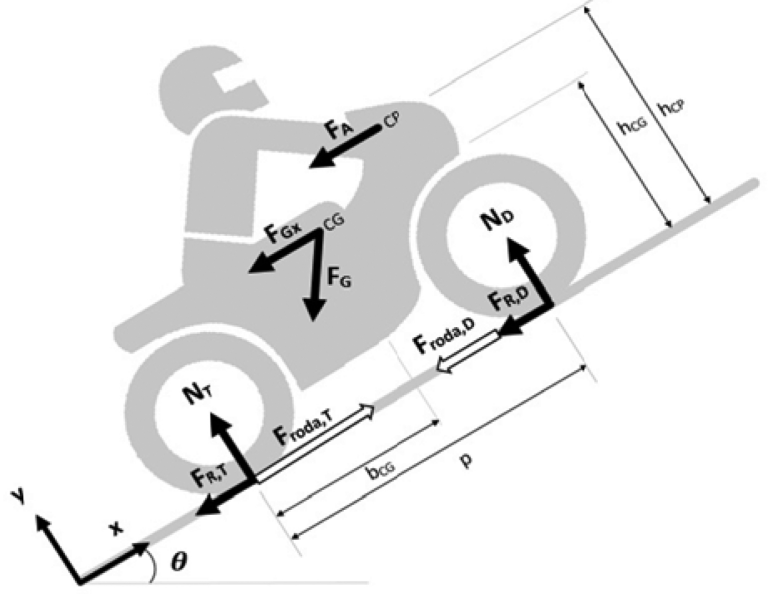

Figura 1: Forças existentes no movimento e frenagem da motocicleta

termos à direita podem ser nulos ou não nulos.

$$
F_{\text {roda }, T}=\alpha \frac{P}{V}-\mu_{T} N_{T}
$$

A potência máxima do motor $(\mathrm{P})$ é uma curva que varia com a rotação, mas essa informação não será importante especificamente neste trabalho já que ou estudamos a motocicleta em curva com velocidade constante $(\mathrm{P}=$ cte $)$ ou durante a frenagem $(\alpha=0)$. No entanto, destacamos que as equações aqui apresentadas são gerais, podendo ser usadas em movimentos mais complexos. O coeficiente de utilização do acelerador $(\alpha)$ pode variar de 0 a 1 (condição em que toda a potência do motor em uma determinada rotação está sendo usada). O coeficiente de utilização do freio $\left(\mu_{T}\right.$, traseiro; ou $\mu_{D}$, dianteiro) varia de 0 a $f$, onde $f$ é o coeficiente de atrito entre o pneu e o piso.

De forma similar, a equação (2) apresenta a força na roda dianteira. Como as motocicletas, com raríssimas exceções, não têm tração dianteira, usamos apenas o termo de frenagem.

$$
F_{\text {roda,D }}=-\mu_{D} N_{D}
$$

As outras forças que atuam sobre a motocicleta tendem a resistir ao movimento, como aquelas apresentadas na equação (3), na qual $F_{A}$ é a resistência aerodinâmica, $F_{R}$ é a resistência à deformação do pneu (resistência de rolagem), e $F_{G x}$ é a componente da força da gravidade na direção do movimento. Em alguns casos particulares essas forças podem assumir valores negativos (i.e., auxiliam o movimento), como 
quando o vento sopra por trás ou nas descidas.

$$
F_{\text {res }}=-F_{A}-F_{R}-F_{G x}
$$

A equação (3) pode ser detalhada pela equação (4), expressando cada força a partir de seus parâmetros.

$$
F_{\text {res }}=-k_{A}(V-W)^{2}-C_{R} m g \cos \theta-m g \sin \theta
$$

O primeiro termo do lado direito da equação (4) é a força aerodinâmica $\left(\mathrm{F}_{\mathrm{A}}\right)$, que depende da velocidade relativa entre a motocicleta $(\mathrm{V})$ e o vento $(\mathrm{W})$, e do fator de arrasto $\left(\mathrm{k}_{\mathrm{A}}\right)$, que, por sua vez, é função da forma do conjunto piloto/motocicleta e da massa especifica do ar. $\mathrm{O}$ fator de arrasto $\mathrm{k}_{\mathrm{A}}$ varia com a velocidade e com a posição do piloto, mas aqui iremos considera-lo constante. O segundo termo representa a resistência de rolagem dos pneus $\left(F_{R}\right)$, que é função do coeficiente de rolagem $\left(\mathrm{C}_{R}\right)$, do peso da motocicleta $(m g)$ e do ângulo da pista $(\theta)$. Em geral, o coeficiente de rolagem varia com a velocidade da motocicleta e com a construção e pressão do pneu, mas neste trabalho consideramos $\mathrm{C}_{\mathrm{R}}$ constante. Finalmente, há a influência do peso $\left(\mathrm{F}_{\mathrm{Gx}}\right)$ no movimento, como indicado pelos últimos termos nas equações (3) e (4).

Os pontos de aplicação das forças resistivas são distintos. A força de arrasto $\left(F_{A}\right)$ é aplicada no centro de pressão $(\mathrm{CP})$, a força da gravidade $\left(F_{G}\right)$ é aplicada no centro de gravidade $(\mathrm{CG})$, e a força de rolagem dos pneus $\left(F_{R}\right)$ é aplicada na interface entre o pneu e a pista, como indicado na Fig. 1. O fato das forças terem pontos diferentes de atuação será importante posteriormente no cálculo dos momentos. Veremos adiante que a inércia da motocicleta $(\mathrm{ma})$, agindo no centro de gravidade combinado piloto/motocicleta, também influencia o movimento. Por exemplo, quanto maior a massa do conjunto, menor será a aceleração para uma mesma força na roda.

Outras duas forças que existem na motocicleta durante a aceleração ou frenagem são as forças normais nas rodas. Essas forças não influenciam diretamente no movimento para frente, mas são importantes no cálculo da aderência. A equação (5) é a normal da roda dianteira, e a equação (6) apresenta a normal traseira. As duas equações são obtidas fazendo um balanço de forças em y e um balanço de momentos em relação ao ponto de contato da roda traseira com o piso.

$$
N_{D}=\frac{F_{G} b_{C G} \cos \theta-F_{A} h_{C P}-m a h_{C G}-F_{G} h_{C G} \sin \theta}{p}
$$

$$
N_{T}=m g \cos \theta-N_{D}
$$

Interessante observar nas equações (5) e (6) que as normais não dependem apenas do projeto da motocicleta (massa e geometria) e da pista (inclinação), mas também do próprio movimento da motocicleta. Isto é, quando há uma aceleração $(a)$ positiva, por exemplo, há uma transferência de peso para a roda traseira. Já na frenagem, é a roda dianteira que tem a normal aumentada. Outra característica do movimento que pode interferir na normal é a velocidade da moto, que influencia a força aerodinâmica (aplicada no centro de pressão).

A equação (7) é a Segunda Lei de Newton aplicada ao movimento da motocicleta na direção $\mathrm{x}$. No caso sem a utilização dos freios $\left(\mu_{D}=\mu_{T}=0\right)$, se as forças resistivas forem menores (em módulo) que a força na roda traseira, a moto vai acelerar. Se as forças resistivas forem maiores que a força na roda traseira, a moto irá diminuir a sua velocidade. No caso geral, o balanço (positivo ou negativo) entre as forças na direção $x$ depende se o piloto está acelerando $(\alpha>0)$, se está freando $\left(\mu_{D}>0\right.$ e/ou $\left.\mu_{T}>0\right)$, se há declive na pista $(\theta \neq 0)$, e se há velocidade relativa do vento $(V-W \neq 0)$, bem como dos parâmetros do conjunto piloto/motocicleta, dados por $k_{A}, C_{R}$ e $m$

$$
m a=F_{\text {roda }, D}+F_{\text {roda }, T}+F_{\text {res }}
$$

Sabemos que a aceleração é a derivada da velocidade com o tempo, resultando então na equação (8) - uma equação diferencial em $\mathrm{V}$ que neste trabalho é resolvida pelo método das diferenças finitas.

$$
\begin{aligned}
& m a=m \frac{d V}{d t}=\alpha \frac{P}{V}-\mu_{T} N_{T}-\mu_{D} N_{D} \\
& -k_{A}(V-W)^{2}-C_{R} m g \cos \theta-m g \sin
\end{aligned}
$$

A solução computacional da equação diferencial (8), ao invés de uma solução analítica, é justificada para o caso geral porque $\mathrm{P}, \mathrm{N}_{\mathrm{D}}$ e $\mathrm{N}_{\mathrm{T}}$ são funções não lineares da velocidade. No caso particular de $\mathrm{P}$, a solução é mais complicada ainda já que a relação entre $\mathrm{P}$ e $\mathrm{V}$ depende também da marcha escolhida pelo piloto, o que em geral depende da história da velocidade, não apenas do seu valor momentâneo.

No caso de curva, a força centrípeta não influencia diretamente o movimento para frente, mas, como ela usa parcialmente o atrito disponível, acaba limitando indiretamente a força que pode ser aplicada na roda tanto para aceleração quanto para frenagem. A força centrípeta aplicada sobre a moto 
é apresentada pela equação (9). Mais para frente iremos dividir essa força centrípeta entre as duas rodas.

$$
F_{\text {cent }}=\frac{m V^{2}}{R}
$$

\subsection{Perda de aderência das rodas dianteira e traseira}

Considerando o movimento mais geral, i.e, durante uma curva, a força de atrito máxima entre um dos pneus e a pista (dada pelo coeficiente de atrito multiplicado pela normal $f N_{i}$ ) não pode ser superada pela força resultante composta pela força longitudinal na roda (equações 1 ou 2) e pela força centrípeta (equação 9), conforme mostrado na Fig. 2.

O limite de uso do atrito é representado pela equação (10), onde o índice i é usado para indicar que a equação vale tanto para a dianteira $(\mathrm{i}=\mathrm{D})$ quanto para traseira $(\mathrm{i}=\mathrm{T})$.

$$
F_{\text {roda }, i}^{2}+F_{\text {cent }, i}^{2} \leq\left(f N_{i}\right)^{2}
$$

O coeficiente de atrito $(f)$ depende do material da pista, da construção e da calibragem do pneu, bem como das condições da pista (e.g., pista seca, pista molhada, pista suja, lama). Por exemplo, em pista seca o valor típico do coeficiente é 0,8 , e, em pista úmida, 0,4 [8]. Caso a condição da equação (10) não seja satisfeita, ocorrem fenômenos indesejáveis. Nas curvas, a moto perde a aderência e sai pela tangente. Nas retas, o pneu desliza, provocando uma diminuição no coeficiente de atrito (que passa de atrito estático para dinâmico) e diminuindo drasticamente a estabilidade da motocicleta $[8,10]$.

Ainda analisando a motocicleta na curva, mas agora a observando frontalmente (Fig. 3), vemos as forças que atuam no plano $y z$. Para que haja o equilíbrio entre a normal e o peso em y, e entre a força centrípeta (atrito) e a centrífuga (inércia radial), a moto precisa permanecer em um ângulo de rolagem $\phi$. Na prática, esse valor é limitado pela

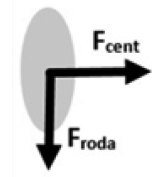

Figura 2: Vista superior da área de contato, no plano $x z$, entre o pneu traseiro deformado da motocicleta e a estrada, apresentando a força centrípeta e força aplicada na roda.

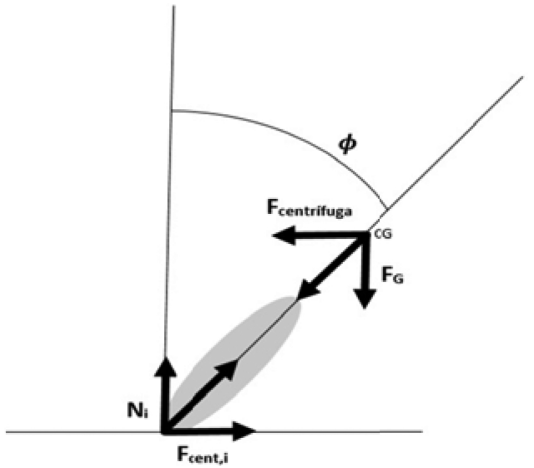

Figura 3: Vista frontal da motocicleta, no plano $y z$, apresentando as forças aplicadas no pneu e no centro de gravidade.

geometria da pedaleira, em geral com valor máximo de $50^{\circ}$ [8]. A razão pela qual os pilotos profissionais se inclinam em direção da pista durante as curvas é para conseguir baixar o centro de gravidade (do conjunto piloto/motocicleta) sem que a pedaleira toque no chão. Neste trabalho, consideramos constante o centro de gravidade do conjunto.

\subsection{Velocidade máxima na curva}

A velocidade máxima que a motocicleta pode permanecer em uma curva, em velocidade constante, sem perder a aderência, pode ser obtida substituindo a equação (9) na equação (10). A distribuição da força centrípeta entre as duas rodas é feita a partir do balanço dos momentos dessas duas forças radiais em relação a um eixo na direção $z$ que passa pelo centro de gravidade. Explicitando a velocidade, resultam as equações (11) para a roda dianteira e (12) para a traseira:

$$
\begin{gathered}
V_{D}^{2} \leq\left(\frac{p}{b_{C G}} \frac{R}{m}\right) \sqrt{\left(f N_{D}\right)^{2}-F_{\text {roda }, D}^{2}} \\
V_{T}^{2} \leq\left(\frac{p-b_{C G}}{b_{C G}} \frac{R}{m}\right) \sqrt{\left(f N_{T}\right)^{2}-F_{\text {roda }, T}^{2}}
\end{gathered}
$$

Vemos que tanto o coeficiente de atrito $(f)$ quanto o raio da curva $(R)$ são importantes para a velocidade máxima de curva. Quanto menor o atrito e menor o raio de curvatura, menor será a velocidade máxima possível. Importante notar que a força na roda (equação 7) depende do arrasto aerodinâmico (equação 4), que por sua vez depende da velocidade da motocicleta. Logo, no caso geral, as equações (11) e (12) necessitam de uma solução numérica para a obtenção da velocidade máxima de curva.

A diferença entre a roda dianteira na equação (11) e a traseira na equação (12) é causada pela 
utilização do acelerador e dos manetes de freio (casos em que $\left.\mathrm{F}_{\text {roda, } \mathrm{D}} \neq \mathrm{F}_{\text {roda, } \mathrm{T}}\right)$ e da distribuição da força centrípeta entre as duas rodas, que depende da posição do centro de gravidade. Neste trabalho, escolhemos como velocidade máxima para que a motocicleta consiga realizar a curva o menor valor obtido entre as equações (11) e (12).

No caso de aceleração nula, vemos pela equação (7) que a força nas duas rodas deve ser igual à força resistiva, calculada pela equação (4). Portanto, podemos ver que quanto maiores forem as resistências, maior terá que ser a força na roda e, consequente, menor a velocidade com que a moto poderá fazer a curva (equação 11 e 12). É importante notar que mesmo com velocidade constante, o piloto em geral usa o acelerador para que a tração equilibre as resistências, ou então usa o freio, no caso de curva em descida.

Na seção de estudos de casos, vamos usar três modelos de curva baseados nas equações (11) e (12). O primeiro é o modelo de curva sem resistência (CSR) em que consideramos $\mathrm{k}_{\mathrm{A}}=\mathrm{C}_{\mathrm{R}}=\theta=0$. Nesse caso, pelas equações (4) e (7), vemos que $\mathrm{F}_{\text {res }}=\mathrm{F}_{\text {roda }}=0$. Então, as equações (11) e (12) se transformam na clássica equação (13) [8].

$$
V_{D, T}^{2} \leq f R g\left\{\begin{array}{l}
\alpha=0 \\
\mu_{D}=\mu_{T}=0 \\
F_{r e s}=0
\end{array}\right.
$$

No segundo modelo de curva (CRC) consideramos que a resistência aerodinâmica é constante, i.e., usamos uma velocidade de referência para o cálculo de $F_{\text {res }}$ na equação (4), o que permite calcular $\mathrm{V}$ explicitamente nas equações (11) e (12), já que agora $\mathrm{F}_{\text {roda }}$ não varia com a velocidade.

O terceiro modelo de curva (CRV), com resistência variável, usa a velocidade real da motocicleta para o cálculo de $F_{\text {res }}$, obrigando que as equações (11) e (12) sejam resolvidas iterativamente, uma vez que $\mathrm{F}_{\text {roda }}\left(=\mathrm{F}_{\text {res }}\right)$ é função da velocidade por causa do arrasto aerodinâmico.

\subsection{Desaceleração e distância de frenagem}

A desaceleração da motocicleta é calculada pela equação (8), que é a equação (7) alimentada pelas equações (1), (2) e (4). Durante a frenagem, assim como no caso da curva em velocidade constante, consideramos três modelos distintos: sem resistência, resistência constante e resistência variável. Para o modelo de frenagem sem resistência (FSR) e com resistência constante (FRC), consideramos que as forças resistivas (arrasto aerodinâmico em particular) não são função da velocidade, e, portanto, a equação (7) se transforma em uma equação algébrica simples para o cálculo da aceleração. No caso do modelo de frenagem com resistência variável (FRV), a variação da velocidade durante a frenagem implica em uma variação da resistência aerodinâmica, fazendo com que a equação (8) seja uma equação diferencial (aqui resolvida por diferenças finitas).

Considerando o caso específico em que o acelerador não é usado $(\alpha=0)$, que os dois freios são usados com o mesmo coeficiente $\left(\mu_{D}=\mu_{T}\right)$ e que a resistência aerodinâmica é constante (i.e., usando uma velocidade de referência), a equação (8) se transforma na equação (14), que será chamada de modelo de frenagem com resistência constante (FRC).

$$
a=-\mu g \cos \theta+\frac{F_{r e s}}{m}\left\{\begin{array}{l}
\alpha=0 \\
\mu=\mu_{D}=\mu_{T} \\
F_{\text {res }}=\text { cte }
\end{array}\right.
$$

Simplificando mais ainda, desprezando agora as resistências, obtemos a equação 15) (modelo de frenagem sem resistência, FSR).

$$
a=-\mu g\left\{\begin{array}{l}
\alpha=0 \\
\mu_{D}=\mu_{T} \\
F_{r e s}=0
\end{array}\right.
$$

Para os casos FRC, equação (14), e FSR, equação (15), uma vez determinada a desaceleração $(a)$, a distância de frenagem pode ser determinada pela equação (16), classicamente conhecida como Equação de Torricelli.

$$
d_{\text {frenagem }}=\frac{V_{i n i}^{2}-V_{f i n}^{2}}{-2 a} a=c t e
$$

No caso do modelo FRV, em que o valor da aceleração não é constante, a distância de frenagem é calculada resolvendo a equação (8) por diferenças finitas.

Um exemplo de frenagem pode ser visto na Fig. 4, para o caso em que a motocicleta está com velocidade de cruzeiro de $100 \mathrm{~km} / \mathrm{h}$ e reduz para $60 \mathrm{~km} / \mathrm{h}$, sendo a força de frenagem utilizada ao máximo $\left(\mu_{\mathrm{D}}=\mu_{\mathrm{T}}=f=0,8\right)$. Temos que, para o caso sem resistência (FSR), a desaceleração é constante com valor de $0,8 \mathrm{~g}$, até tornar-se nula ao atingir os desejados $60 \mathrm{~km} / \mathrm{h}$, quando então o piloto desativa o freio. Já para o caso com resistência constante (FRC), a desaceleração é de $0,93 \mathrm{~g}$. Para o caso com 


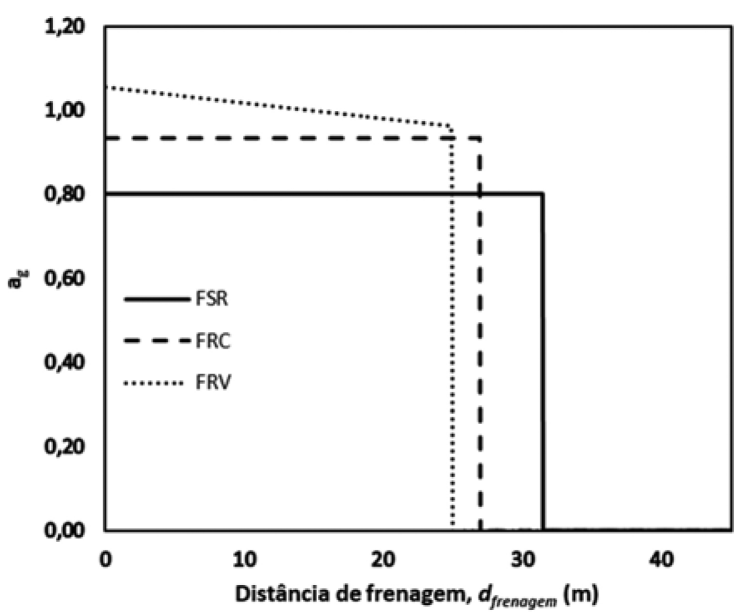

Figura 4: Desaceleração na frenagem em função da distância. $\mu_{D}=\mu_{T}=f=0,8$. Redução de velocidade de $100 \mathrm{~km} / \mathrm{h}$ para $60 \mathrm{~km} / \mathrm{h}$. São considerados o modelo sem resistência (FSR), resistência constante (FRC) e resistência variável (FRV).

resistência variável (FRV), o valor da desaceleração varia de $1,06 \mathrm{~g}$ a $0,96 \mathrm{~g}$, já que quando a velocidade diminui, diminui também o arrasto aerodinâmico que auxilia a desaceleração.

Outro exemplo de frenagem pode ser visto na Fig. 5, para o caso de redução de velocidade com coeficiente de utilização do freio de $0,4\left(\mu_{\mathrm{D}}=\mu_{\mathrm{T}}=\right.$ $f=0,4$; o que fisicamente é análogo ao uso de $50 \%$ do freio com coeficiente de atrito de 0,8$)$. Vemos que, para o caso sem resistência (FSR), a desaceleração é constante com valor de $0,32 \mathrm{~g}$. Já para o caso com resistência constante (FRC), a desaceleração é $0,54 \mathrm{~g}$, e para o caso com resistência variável (FRV) a desaceleração fica na faixa $0,66 \mathrm{~g}$ a $0,57 \mathrm{~g}$.

Observa-se então o esperado, que uma menor utilização do freio (por desejo do piloto ou por uma diminuição no coeficiente de atrito entre o pneu e a pista) provoca uma maior distância necessária para a frenagem.

Outra forma de verificar a influência das simplificações nos modelos é fixando a proporção da força do freio dianteiro em relação à força de frenagem total. A Fig. 6 mostra os resultados para o caso em que o motociclista escolhe $\mu_{\mathrm{D}}$ e $\mu_{\mathrm{T}}$ de forma que $70 \%$ da força de frenagem sempre seja proporcionada pela roda dianteira. Por exemplo, se o piloto desejar uma desaceleração de $0,4 \mathrm{~g}$, pelo modelo FRC ele terá que usar $\mu_{\mathrm{T}}=0,22$ e $\mu_{\mathrm{D}}=0,29$. Já pelo modelo FSR, para a mesma frenagem $\left(\mathrm{a}_{\mathrm{g}}=0,4 \mathrm{~g}\right)$ esses valores seriam $\mu_{\mathrm{T}}=0,45$ e $\mu_{\mathrm{D}}=0,38$.
Assim, podemos concluir que, para um mesmo par de coeficientes de utilização do freio $\left(\mu_{\mathrm{T}}\right.$ e $\left.\mu_{\mathrm{D}}\right)$, o modelo com resistência constante (FRC) apresenta valores de frenagem maiores que o modelo sem resistência (FSR). Isso acontece porque as forças resistivas na equação (7) impõem uma desaceleração ao movimento da motocicleta. Neste caso, a desaceleração adicional causada pelas resistências tem valor aproximado de $0,16 \mathrm{~g}$, sendo esse o motivo das curvas do modelo com resistência constante (FRC) não partirem da origem na Fig. 6.

Além da distância de frenagem, que é a distância após o início do acionamento do freio, existe também uma distância percorrida entre o momento em que o piloto observa a placa e o momento em que ele atua sobre o freio da motocicleta. Essa distância é chamada de distância de reação do piloto, e é calcu-

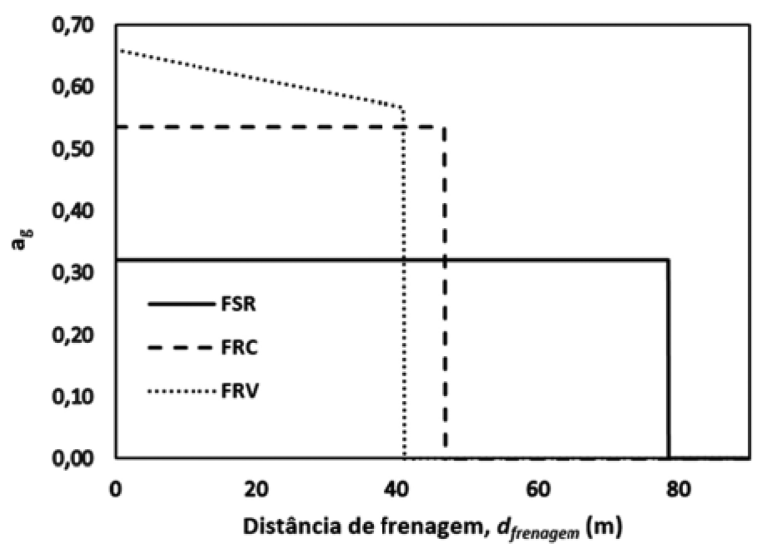

Figura 5: Desaceleração na frenagem em função da distância. $\mu_{D}=\mu_{T}=f=0,4$. Redução de velocidade de $100 \mathrm{~km} / \mathrm{h}$ para $60 \mathrm{~km} / \mathrm{h}$. São considerados o modelo sem resistência (FSR), resistência constante (FRC) e resistência variável (FRV).

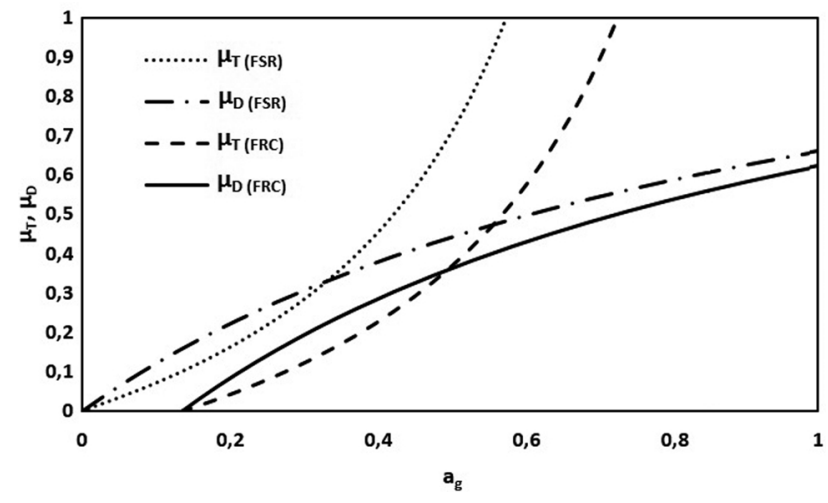

Figura 6: Uso dos freios dianteiro e traseiro em função da desaceleração desejada. Casos sem resistência (FSR) e resistência constante (FRC) utilizando $70 \%$ da força do freio dianteiro. 
lada pela equação (17), levando em conta o tempo de reação $\delta t$ e a velocidade de cruzeiro $\left(\mathrm{V}_{\text {cruz }}\right)$ em $\mathrm{m} / \mathrm{s}$. O CONTRAN [5] considera nos seus cálculos $\delta t=2,5$ segundos.

$$
d_{\text {reação }}=\delta t V_{\text {cruz }}
$$

\subsection{Limite de stoppie e técnica de frenagem}

Durante a frenagem, um dos limites para que o piloto fique em segurança é o limite de stoppie, que é a perda de contato da roda traseira com o chão. Esse limite pode ser determinado calculando o momento em que a normal traseira fica nula na equação (6). Se desconsiderarmos as resistências e considerarmos a mesma utilização dos dois freios, esse limite é expresso pela equação (18), na qual vemos que o valor máximo do coeficiente de utilização do freio para que não ocorra o stoppie é maior no caso de motos com menor altura do centro de gravidade $\left(\mathrm{h}_{\mathrm{CG}}\right)$ ou com centro de gravidade mais deslocado para a traseira (menor valor de $b_{G}$, Fig. 1).

$$
\mu=\frac{p-b_{C G}}{h_{C G}}\left\{\begin{array}{l}
\alpha=0 \\
\mu_{D}=\mu_{T} \\
F_{r e s}=0
\end{array}\right.
$$

Uma forma alternativa de representar a atuação dos freios é a partir da proporção da força do freio dianteiro $\left(\mathrm{P}_{\mathrm{D}}\right)$. Por exemplo, considerando um coeficiente de atrito unitário $(f=1)$, digamos que uma motocicleta apresenta $1000 \mathrm{~N}$ de força de atrito disponível na roda dianteira $\left(f . \mathrm{N}_{\mathrm{D}}\right)$ e $600 \mathrm{~N}$ na roda traseira $\left(f . \mathrm{N}_{\mathrm{T}}\right)$. No entanto, durante a frenagem, são utilizados $800 \mathrm{~N}$ de força de frenagem na roda dianteira e $420 \mathrm{~N}$ na traseira. O coeficiente de utilização do freio $(\mu)$ é calculado pela razão da força utilizada e da força disponível na roda, i.e., $\mu_{D}=0,8(=800 / 1000)$ e $\mu_{T}=0,7(=420 / 600)$. Já a proporção do freio dianteiro $\left(\mathrm{P}_{\mathrm{D}}\right)$ é calculada pela razão da força utilizada na roda dianteira $(800 \mathrm{~N})$ e a força total utilizada $(800+420=1220 \mathrm{~N})$, resultando em $\mathrm{P}_{\mathrm{D}}=0,66 \mathrm{~A}$ atuação dos freios pode então ser caracterizada ou pelos dois $\mu$ 's ou por um dos dois $\mu$ 's juntamente com o $\mathrm{P}_{\mathrm{D}}$.

Manipulando algebricamente as equações anteriores, os coeficientes de utilização do freio (dianteiro e traseiro, $\mu_{\mathrm{D}}$ e $\mu_{\mathrm{T}}$ ) se relacionam com a proporção da força do freio dianteiro $\left(\mathrm{P}_{\mathrm{D}}\right)$ e com a aceleração (a) pelas equações (19) e (20), respectivamente:

$$
\begin{gathered}
P_{D}=\frac{\mu_{D}}{\mu_{D}+\mu_{T}\left(\frac{C_{1}-C_{2}}{\frac{M_{r e s}}{m g \cos \theta}-C_{1}}-1\right)} \\
a_{g}=-\frac{a}{g}=\mu_{T} \cos \theta \\
+\left(\frac{\mu_{D}-\mu_{T}}{C_{2}-C_{1}}\right)\left(\frac{M_{\text {res }}}{m g}-C_{1} \cos \theta\right)+\frac{F_{r e s}}{m g}
\end{gathered}
$$

Essas equações dependem do termo resistivo $M_{\text {res }}$, dado pela equação (21):

$$
M_{r e s}=m g h_{C G} \sin \theta+k_{A} h_{c p}(V-W)^{2}
$$

Nas equações (19) e 20 aparecem ainda dois parâmetros, $\mathrm{C}_{1}$ e $\mathrm{C}_{2}$, que são funções da geometria da motocicleta e da utilização dos freios.

$$
\begin{gathered}
C_{1}=\frac{\mu_{T} h_{C G}+b_{C G}}{p-b_{C G}} \\
C_{2}=\frac{\mu_{D} h_{C G}}{p-b_{C G}}
\end{gathered}
$$

No caso $\mu_{D}=\mu_{T}$, considerando a força de resistência constante, a equação 20 torna-se a equação (14) No caso de resistência nula, torna-se a equação (15).

Já a equação 19 transforma-se na equação (24) para o caso sem resistência e com valores de coeficientes de utilização de freio distintos.

$$
P_{D}=\frac{\mu_{D}\left(b_{C G}+h_{C G} \mu_{T}\right)}{p \mu_{T}+b_{C G}\left(\mu_{D}-\mu_{T}\right)}\left\{F_{r e s}=M_{r e s}=0\right.
$$

\subsection{Metodologia de estudo da velocidade máxima em curva e de frenagem em linha reta}

Para estudar todo o processo de curva de uma motocicleta, analisamos o caso apresentado na Fig. 7. A motocicleta está em velocidade de cruzeiro $V_{\text {cruz }}$ no trecho 1. No início do trecho 2 o piloto percebe a placa. Levando em conta o tempo de reação, somente no início do trecho 3 é que o piloto inicia a frenagem, até alcançar a velocidade da curva no início do trecho 4.

Considerando a manobra completa da Fig. 7, queremos estudar (1) a distância mínima antes do início da curva em que o piloto precisa observar a placa para que tenha tempo de reagir e desacelerar, e (2) o valor da velocidade máxima que deve estar escrito 


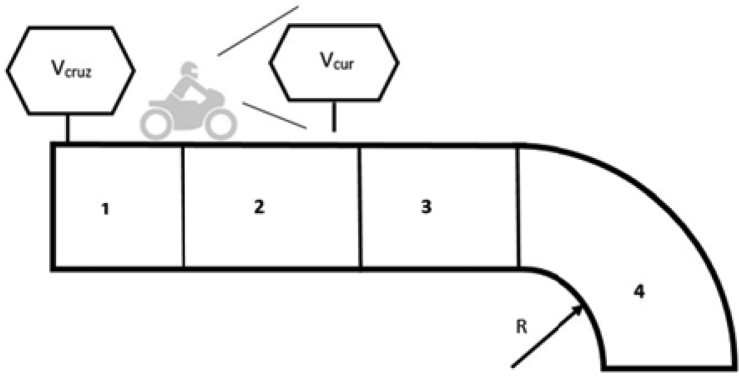

Figura 7: Movimento completo da manobra. Trecho 1: velocidade de cruzeiro; trecho 2: distância para o piloto reagir; trecho 3: frenagem; trecho 4: curva em velocidade constante.

na placa para que a motocicleta consiga manter a aderência durante a curva. Em outras palavras, queremos determinar onde deve estar a placa e qual o valor escrito nela.

A motocicleta utilizada para o estudo tem as características apresentada na Tabela 1.

A pista tem inclinação $(\theta)$ de $5^{\circ}$ e o vento tem velocidade $(\mathrm{W})$ contrária de $-5 \mathrm{~km} / \mathrm{h}$. No trecho 1 , a motocicleta tem velocidade de cruzeiro $\left(\mathrm{V}_{\text {cruz }}\right)$ de $100 \mathrm{~km} / \mathrm{h}$. Essa velocidade irá interferir na distância de frenagem, como apresentado na equação (16). Já no início do trecho 2 , o piloto visualiza a placa de redução, levando uma certa distância até que inicie a frenagem, como calculada pela equação (17).

No trecho 3, com o manete de freio já acionado, a motocicleta sofre a desaceleração. Essa reação de frenagem é calculada utilizando as equações de desaceleração, considerando ainda os limites de stoppie e de perda de aderência. Nesta análise, usamos o modelo de frenagem com força de resistência constante (FRC). Essa escolha foi feita porque permite o cálculo da dinâmica da moto sem a necessidade da solução da equação diferencial, equação (8), ao mesmo tempo em que apresenta resultados pareci-

Tabela 1: Características físicas e geométricas da motocicleta

\begin{tabular}{ll}
\hline Características & \\
\hline $\mathrm{m}$ & $230 \mathrm{~kg}$ \\
$\mathrm{P}$ & $22,06 \mathrm{~kW}$ \\
$\mathrm{k}_{\mathrm{A}}$ & $0,4 \mathrm{~kg} / \mathrm{m}$ \\
$\mathrm{C}_{\mathrm{R}}$ & 0,02 \\
$\mathrm{~h}_{\mathrm{CG}}$ & $0,75 \mathrm{~m}$ \\
$\mathrm{~h}_{\mathrm{CP}}$ & $0,9 \mathrm{~m}$ \\
$\mathrm{p}$ & $1,4 \mathrm{~m}$ \\
$\mathrm{~b}_{\mathrm{CG}}$ & $0,73 \mathrm{~m}$ \\
\hline
\end{tabular}

dos com o que obteríamos com aquele modelo mais completo (Fig. 4 e 5).

Por fim, no trecho 4, a motocicleta deve apresentar velocidade menor ou igual à velocidade máxima possível (equações 11, 12 e 13) permitida para fazer a curva sem perder a aderência.

\section{Resultados e Discussões}

A análise do movimento da motocicleta será apresentada no sentido inverso ao movimento, partindo do trecho 4 (cálculo da velocidade máxima na curva) e finalizando no trecho 2 (distância de percepção da placa). Nesses estudos, consideramos a influência da pista (coeficiente de atrito, raio de curvatura) e da técnica de frenagem usada pelo piloto. Todas as equações estão escritas no SI. No entanto, alguns gráficos e tabelas apresentam os resultados transformados para unidades mais comuns (e.g., $\mathrm{km} / \mathrm{h}$ ).

\subsection{Velocidade máxima na curva e raio de curvatura mínimo}

A Tabela 2 apresenta a velocidade máxima da motocicleta na curva em função do raio de curvatura e do coeficiente de atrito $f(0,4$ para pista molhada; 0,8 para pista seca). A velocidade máxima é dada pela equação (13) desconsiderando as resistências, e pelo mínimo entre as equações (11) e (12) quando consideramos as resistências.

Pelo modelo sem resistência (CSR), a velocidade máxima na curva pode ser maior que nos modelos com resistência, pois a motocicleta não precisa de força na roda para vencer as forças contrárias ao movimento, sobrando mais do atrito para a força centrípeta. Para o modelo sem resistência, o limite de velocidade é o mesmo para as duas rodas $(\mathrm{D}, \mathrm{T})$. No caso dos modelos com resistência (constante,

Tabela 2: Velocidade máxima de entrada na curva em função do raio de curvatura e do coeficiente de atrito. Modelos sem resistência (CSR), resistência constante (CRC) e resistência variável (CRV).

\begin{tabular}{lllll}
\hline $\mathrm{R}[\mathrm{m}]$ & $f$ & & $\mathrm{~V}[\mathrm{~km} / \mathrm{h}]$ \\
\hline \multirow{2}{*}{340} & & $\mathrm{CSR}$ & $\mathrm{CRC}$ & $\mathrm{CRV}$ \\
\hline \multirow{2}{*}{200} & 0,4 & $132^{(\mathrm{D}, \mathrm{T})}$ & $123^{(\mathrm{D})}$ & $87^{(\mathrm{T})}$ \\
\cline { 2 - 5 } & 0,8 & $186^{(\mathrm{D}, \mathrm{T})}$ & $174^{(\mathrm{D})}$ & $139^{(\mathrm{D})}$ \\
\cline { 2 - 5 } 110 & 0,4 & $101^{(\mathrm{D}, \mathrm{T})}$ & $94^{(\mathrm{D})}$ & $79^{(\mathrm{T})}$ \\
\cline { 2 - 5 } & 0,8 & $143^{(\mathrm{D}, \mathrm{T})}$ & $133^{(\mathrm{D})}$ & $116^{(\mathrm{D})}$ \\
\cline { 2 - 5 } & 0,4 & $75^{(\mathrm{D}, \mathrm{T})}$ & $70^{(\mathrm{D})}$ & $65^{(\mathrm{T})}$ \\
\hline
\end{tabular}


CRC, ou variável, CRV), as velocidades máximas são diferentes para as duas rodas. Na Tabela 2, a roda que perde primeiro a aderência, e que portanto limita a velocidade da moto, é indicada no índice superior (D ou T). Por exemplo, para o modelo CRV, a moto sempre perde a traseira em pista molhada $(f=0,4)$ e perde a dianteira em pista seca $(f=0,8)$, para qualquer raio de curvatura. Já no caso CRC, a moto estudada sempre é limitada pela perda de aderência na dianteira.

Em geral, como esperado, quanto menor o coeficiente de atrito e menor o raio de curvatura, menor a velocidade máxima na curva. Outro padrão na Tabela 2 é que o modelo CRV é mais conservativo que o modelo CRC, que por sua vez é mais conservativo que o CSR.

A Tabela 3 apresenta a mesma análise, mas agora especificando a velocidade desejada e calculando o raio mínimo de curvatura permitido para cada condição. Para o modelo CRV, no caso de baixo atrito $(f=0,4)$, a moto não tem aderência a $100 \mathrm{~km} / \mathrm{h}$ mesmo em linha reta, por isso o valor do raio de curvatura mínimo não é indicado na Tabela 3.

\subsection{Limite na frenagem: stoppie}

Agora passamos a estudar o Trecho 3, analisando primeiramente em que caso o limite da frenagem se dá por travamento nas rodas ou por stoppie (perda do contato da roda traseira com o chão). Neste caso, usamos o modelo com resistência constante (FRC) e o mesmo coeficiente de utilização do freio nas duas rodas $\left(\mu=\mu_{\mathrm{D}}=\mu_{\mathrm{T}}\right)$. A equação (14) é usada para o cálculo da (des)aceleração, e as equações (5) e (6) para o cálculo da normal traseira. A Fig. 8 mostra que, até limite $\mu=0,92$ (ponto $\mathrm{A}$ ), não há levantamento da roda traseira $\left(\mathrm{N}_{\mathrm{T}} \leq 0\right)$. Logo o limite da frenagem é dado pela perda de aderência. Caso o coeficiente de utilização do freio seja maior que 0,92

Tabela 3: Raio mínimo em função da velocidade e do coeficiente de atrito. Modelos sem resistência (CSR), resistência constante $(C R C)$ e resistência variável (CRV).

\begin{tabular}{lllll}
\hline $\mathrm{V}(\mathrm{km} / \mathrm{h})$ & $f$ & \multicolumn{3}{c}{$\mathrm{R}(\mathrm{m})$} \\
\hline \multirow{2}{*}{100} & & $\mathrm{CSR}$ & $\mathrm{CRC}$ & $\mathrm{CRV}$ \\
\hline \multirow{2}{*}{80} & 0,4 & $196^{(\mathrm{D}, \mathrm{T})}$ & $226^{(\mathrm{D})}$ & - \\
\cline { 2 - 5 } & 0,8 & $98^{(\mathrm{D}, \mathrm{T})}$ & $113^{(\mathrm{D})}$ & $137^{(\mathrm{D})}$ \\
\cline { 2 - 5 } & 0,4 & $125^{(\mathrm{D}, \mathrm{T})}$ & $144^{(\mathrm{D})}$ & $216^{(\mathrm{T})}$ \\
\hline \multirow{2}{*}{60} & 0,8 & $62^{(\mathrm{D}, \mathrm{T})}$ & $72^{(\mathrm{D})}$ & $80^{(\mathrm{D})}$ \\
\cline { 2 - 5 } & 0,4 & $71^{(\mathrm{D}, \mathrm{T})}$ & $81^{(\mathrm{D})}$ & $89^{(\mathrm{T})}$ \\
\hline
\end{tabular}

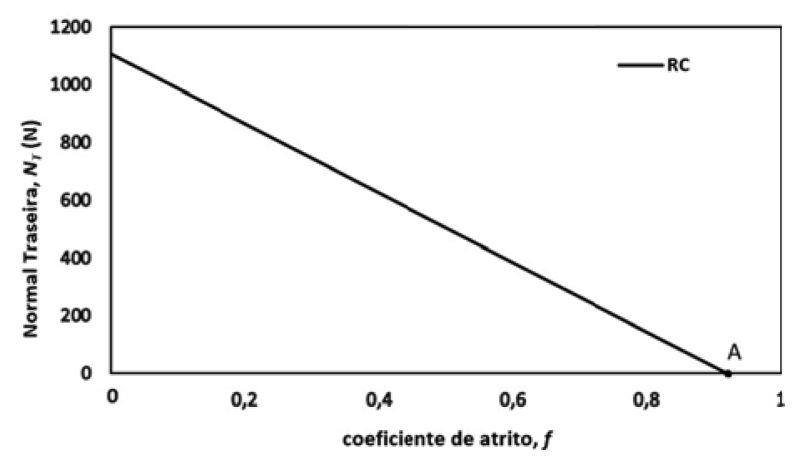

Figura 8: Variação da normal traseira em função do coeficiente de utilização do freio $\left(\mu_{D}=\mu_{T}=\mathrm{f}\right)$. Modelo com resistência constante (FRC) em uma redução de $100 \mathrm{~km} / \mathrm{h}$ a $60 \mathrm{~km} / \mathrm{h}$.

(desde que o atrito permita), a moto irá girar para frente (stoppie) antes de perder a aderência Quando ocorre o stoppie, não há mais sentido em falar em $\mu_{T}$, já que agora a normal traseira é nula.

\subsection{Influência da técnica de frenagem na desaceleração}

Até este momento, todas as análises (com exceção da Fig. 6) foram feitas considerando $\mu_{D}=\mu_{T}$. A partir de agora, iremos estudar a influência da técnica de frenagem na pilotagem. A Fig. 9 apresenta as desacelerações que são obtidas para cada par $\left(\mu_{D}, \mu_{T}\right)$ pela equação 20 . São indicados, também, os valores $\mathrm{P}_{\mathrm{D}}$ (Equação 19) para cada situação. Cossalter [8] apresenta uma figura parecida com a Fig. 9, mas para o caso sem resistência e geometria um pouco diferente.

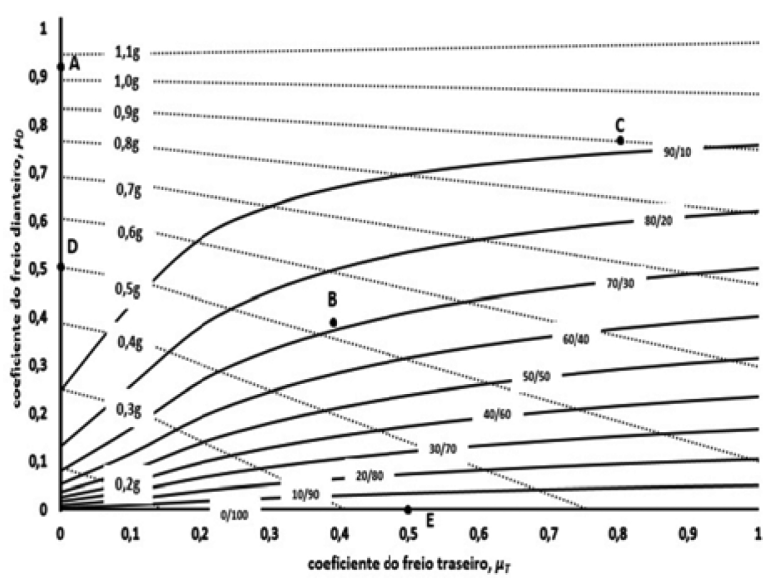

Figura 9: Curvas de desaceleração e distribuição de frenagem $\left(P_{\mathrm{D}} / \mathrm{P}_{\mathrm{T}}\right)$. Modelo com resistência constante (FRC) para uma redução de $100 \mathrm{~km} / \mathrm{h}$ para $60 \mathrm{~km} / \mathrm{h}$. 
O ponto A é o mesmo apresentado na Fig. 7 e afirma que para valores $\mu_{D}=0,92$ e $\mathrm{P}_{\mathrm{D}}=1$ (i.e., curva coincidente com o eixo y; $\mu_{T}=0$ ) a motocicleta apresenta a desaceleração de $1,05 \mathrm{~g}$.

No ponto $\mathrm{B}$, temos os valores de $\mu_{D}=\mu_{T}=$ 0,4 , que corresponde a uma frenagem máxima em pista úmida $(f=0,4)$ ou a utilização de $50 \%$ do freio em pista seca $(f=0,8)$. Para esse caso, $\mathrm{P}_{\mathrm{D}}=0,72$, e a desaceleração é de $0,53 \mathrm{~g}$.

Quando a motocicleta utiliza $100 \%$ da capacidade de frenagem em pista seca $\left(f=\mu_{D}=\mu_{T}=\right.$ $0,8)$, ela consegue uma desaceleração de $0,93 \mathrm{~g}$, com $\mathrm{P}_{\mathrm{D}}=0,92$, como mostra o ponto $\mathrm{C}$.

Os pontos $\mathrm{D}$ e $\mathrm{E}$ mostram a diferença entre o uso de apenas um dos freios (no caso $\mu=0,5$ ). Para o uso único do dianteiro (Ponto $\mathrm{D}, \mu_{\mathrm{D}}=0,5, \mathrm{P}_{\mathrm{D}}=1$ ), a desaceleração é de $0,5 \mathrm{~g}$. Já usando apenas o freio traseiro (Ponto E, $\mu_{\mathrm{T}}=0,5, \mathrm{P}_{\mathrm{D}}=0$ ), a desaceleração é bem menor, $0,35 \mathrm{~g}$. O freio dianteiro permite uma frenagem melhor porque, ao desacelerar, há uma transferência de peso para a roda dianteira, o que aumenta a normal e, consequentemente, a força de atrito disponível. No entanto, embora o travamento de qualquer uma das rodas seja perigoso, o travamento da roda dianteira é bem mais desestabilizante para movimento [10], merecendo bastante cuidado por parte do piloto.

\subsection{Técnica ótima para distância de frenagem mínima}

Na Fig. 10, podemos escolher a técnica de frenagem que possibilita a menor distância de frenagem. Os pontos analisados são os mesmos da Fig. 9. Na Fig. 10, a linha contínua mostra a distância mínima de frenagem para cada uso de freio $\left(\mu_{D}=\mu_{T}=f\right)$. A linha tracejada mostra a qual deve ser a distribuição das forças, $\mathrm{P}_{\mathrm{D}}$, para aquela frenagem ótima.

Para o ponto B, caso de pista úmida $\left(\mu_{D}=\mu_{T}=\right.$ $0,4)$, a distância mínima de frenagem que a motocicleta deve ter para reduzir a velocidade de $100 \mathrm{~km} / \mathrm{h}$ a $60 \mathrm{~km} / \mathrm{h}$ é aproximadamente $47 \mathrm{~m}$. Já no ponto $\mathrm{C}$, caso de pista seca $\left(\mu_{D}=\mu_{T}=0,8\right)$, a distância mínima de frenagem necessária é aproximadamente $27 \mathrm{~m}$. O valor de $\mathrm{P}_{\mathrm{D}}$ ótimo para o caso de pista úmida é 0,72 , enquanto no caso de pista seca é 0,92 . Ou seja, quanto maior o coeficiente de atrito maior a importância relativa do freio dianteiro. Observamos então que não é possível recomendar uma técnica única de frenagem para todos os casos.

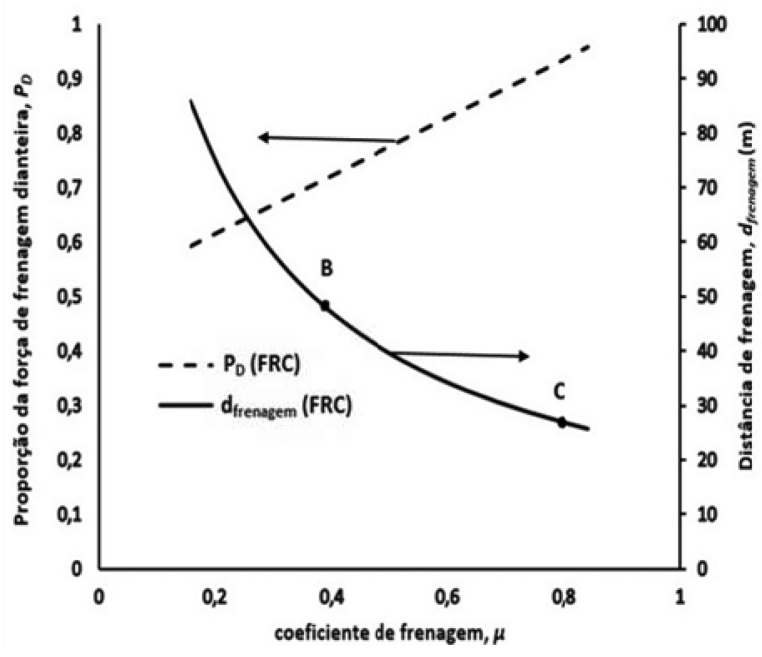

Figura 10: Distância de frenagem mínima e $\mathrm{P}_{\mathrm{D}}$ para $\mu_{D}=\mu_{T}=f$. Modelo de resistência constante (FRC) para redução de $100 \mathrm{~km} / \mathrm{h}$ para $60 \mathrm{~km} / \mathrm{h}$.

\subsection{Influência da técnica na distância de frenagem}

A Fig. 11 apresenta a distância de frenagem para várias distribuições de força de frenagem, $\mathrm{P}_{\mathrm{D}}$, considerando pista seca $(f=0,8)$ e pista úmida $(f=0,4)$. Nas duas curvas percebe-se um ponto de mínimo (frenagem ótima), à esquerda do qual a frenagem é limitada pela perda de aderência da roda traseira e à direita pela roda dianteira. Duas observações são importantes. Primeiro, como já discutido, o valor ótimo de $\mathrm{P}_{\mathrm{D}}$ depende do coeficiente de atrito. Segundo, uma técnica incorreta de frenagem pode significar uma distância muito maior para a dimi-

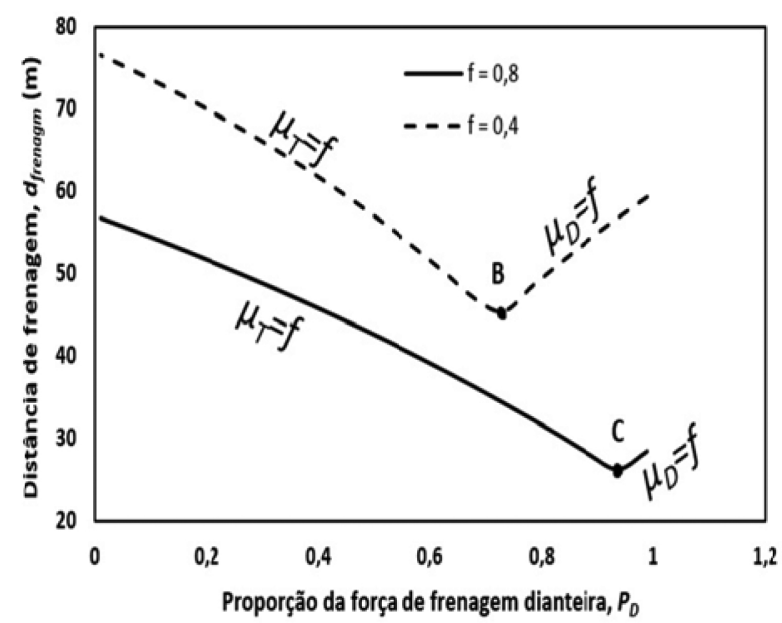

Figura 11: Distância mínima de frenagem em função do $\mathrm{P}_{\mathrm{D}}$ para $f=0,4$ e $\mathrm{f}=0,8$. Modelo de resistência constante (FRC) para redução de $100 \mathrm{~km} / \mathrm{h}$ para $60 \mathrm{~km} / \mathrm{h}$. 
nuição da velocidade. Na Fig. 11 estão indicados os casos $\mathrm{B}$ e C (ótimos) que já haviam aparecido nas Figs. 9 e 10.

\subsection{Distância da placa}

Nesta subseção estudamos o posicionamento da placa de velocidade. Consideramos que o piloto, inicialmente em velocidade de cruzeiro de $100 \mathrm{~km} / \mathrm{h}$, avista a placa indicando a existência de uma curva. O raio de curvatura neste caso é de $110 \mathrm{~m}$. Então, pela Tabela 2, sabemos que $60 \mathrm{~km} / \mathrm{h}$ é uma velocidade segura tanto com baixo quanto com alto atrito. Pelo modelo CRV, que é o mais conservativo, a moto sairia de traseira apenas se estivesse acima de 65 $\mathrm{km} / \mathrm{h}$ com $f=0,4$, e sairia de frente com $f=0,8$ somente acima de $92 \mathrm{~km} / \mathrm{h}$.

Para essa condição $\left(V_{\text {cruz }}=100 \mathrm{~km} / \mathrm{h}=27,8 \mathrm{~m} / \mathrm{s}\right)$, utilizando a equação (17) para calcular a reação do piloto, a distância percorrida até o início da frenagem é de $69 \mathrm{~m}$.

Para avaliar a distância de frenagem da motocicleta, consideramos duas situações: $\mu=0,4$ (que pode significar $100 \%$ do freio em pista úmida ou $50 \%$ do freio em pista seca) e $\mu=0,8$ ( $100 \%$ do freio em pista seca). As distâncias são retiradas da Fig. 10 $\left(\mu_{\mathrm{D}}=\mu_{\mathrm{T}}=\mu\right)$.

A Tabela 4 mostra a distância de reação do piloto, a distância de frenagem da motocicleta, e a soma das duas, que é a distância mínima na qual a placa precisa ser percebida. Para as duas condições de atrito estudadas, consideramos que o piloto ajusta o uso do freio $\left(\mathrm{P}_{\mathrm{D}}\right)$ de forma a obter a melhor frenagem possível $\left(\mu_{\mathrm{D}}=\mu_{\mathrm{T}}=\mu=f\right)$.

Para essa redução de velocidade, o CONTRAN [5] recomenda que a distância de frenagem deve ser considerada $123 \mathrm{~m}$ (usando Torricelli para uma redução de $100 \mathrm{~km} / \mathrm{h}$ para $60 \mathrm{~km} / \mathrm{h}$ com desaceleração de $2 \mathrm{~m} / \mathrm{s}^{2}$ ) Esse cálculo do CONTRAN [5] corresponde ao nosso modelo de frenagem sem resistência (FSR), equação (16). Portanto, vemos que essa recomendação é segura, principalmente porque o piloto irá observar a placa antes de chegar ao local

Tabela 4: Distância de observação da placa em relação ao início da curva para $\mathrm{P}_{\mathrm{D}}$ ótimo $\left(\mu_{\mathrm{D}}=\mu_{\mathrm{T}}=\mu=f\right)$. Redução $100 \mathrm{~km} / \mathrm{h}$ a $60 \mathrm{~km} / \mathrm{h}$.

\begin{tabular}{lllcl}
\hline $\mathrm{R}$ & $\mu$ & \multicolumn{3}{c}{ Distância $(\mathrm{m})$} \\
\hline \multirow{2}{*}{110} & & Reação & Frenagem & Placa \\
\cline { 2 - 5 } & 0,8 & 69 & 27 & 96 \\
\hline
\end{tabular}

onde está posicionada. O modelo com resistência variável (FRV) apresenta uma distância de frenagem de $25 \mathrm{~m}$, valor bem parecido com o obtido pelo modelo FRC (27 m, Tabela 4). O modelo sem resistência resulta em uma distância de frenagem de $32 \mathrm{~m}$. Portanto, dos três modelos, o modelo sem resistência (FSR) é o mais conservativo.

\section{Conclusões}

O trabalho mostrou algumas considerações que devem ser feitas no cálculo da distância de frenagem e da velocidade máxima de curva. Vimos que, no caso da curva, do ponto de vista da segurança, é importante levar em conta as resistências. Já no caso de frenagem em linha reta, o modelo sem resistência é mais conservativo, podendo ser utilizado. Outro resultado importante é que os modelos com resistência constante ou com resistência variável apresentam resultados parecidos, permitindo o uso do primeiro, que não necessita a solução de uma equação diferencial.

Do ponto de vista físico, como esperado, determinamos que quanto menor o atrito e menor o raio de curvatura, menor a velocidade máxima possível na curva. Ainda estudamos os vários limites durante curvas e frenagens, que podem ser a perda de aderência na roda dianteira, a perda de aderência na roda traseira, ou a perda de contato da roda traseira (stoppie).

Destacamos que o modelo matemático apresentado pode ser usado também no caso de acelerações, desde que seja considerada a variação da potência máxima do motor, $\mathrm{P}$, com a rotação do motor.

Vimos também como a técnica de frenagem (proporção dos freios dianteiro e traseiro, $\mathrm{P}_{\mathrm{D}}$ ) influencia na desaceleração e, consequentemente, na distância de frenagem. Um ponto importante é que a técnica ótima de frenagem depende do coeficiente de atrito, o que desaconselha a recomendação de uma técnica universal para todas as situações.

Por fim, apresentamos uma metodologia para o cálculo da posição e da velocidade máxima de uma placa em função das condições de atrito, do raio de curvatura e da utilização do freio. Esses cálculos demonstraram que a recomendação do CONTRAN [5] é segura.

Os modelos apresentados neste trabalho são introdutórios. Em passos seguintes da carreira de engenheiro de motocicletas, o estudante poderá considerar outros fenômenos importantes. Para tanto, ci- 
tamos alguns trabalhos mais avançados. Corno et al. [11] apresentam uma discussão parecida com a aqui apresentada, levando em conta a transferência de peso, o limite entre a perda de aderência e o stoppie, e a influência do arrasto aerodinâmico. Adicionalmente, usando um pacote comercial de simulação, eles estudam outros fenômenos mais complexos, como a variação do coeficiente de atrito com o deslizamento da roda e a influência do movimento da suspensão na frenagem. Toyofuku et al. [12] mostram os resultados de experimentos de frenagem, comparando pilotos com e sem experiência. Baumann et al. [13] apresentam um método de análise de frenagem durante a curva, manobra que pode ser facilitada no caso de motocicletas elétricas com tração nas duas rodas. Özkan et al. [14] mostram uma dimensão que não foi considerada neste trabalho, que é o comportamento do piloto. Eles estudam erros de tráfego, erros no controle da motocicleta, violações de limite de velocidade, realização de manobras perigosas e o uso de equipamentos de segurança. Vlahogianni et al. [15] discutem os riscos específicos do uso das motocicletas. Em particular, destacamos a seção em que eles apresentam os riscos associados à infraestrutura, que incluem o tipo de piso, geometria das estradas, visibilidade do piloto e perda de controle durante frenagens e curvas.

Um ponto muito importante a destacar é que no cálculo da distância mínima de frenagem e da velocidade máxima de curva, consideramos os casos limites. Na prática, com a exceção de pilotos profissionais, é muito difícil que um piloto consiga conduzir a motocicleta nesses casos extremos. Ainda, mesmo um piloto profissional, no dia a dia, não usa a motocicleta em situação limite nas curvas e frenagens. Outro ponto importante é que os valores calculados dependem fortemente dos parâmetros utilizados, não podendo, portanto, ser utilizados como regras gerais para qualquer caso.

Voltando ao ensino na graduação, exemplos como o aqui apresentado levam cerca de seis horas para serem construídos na disciplina de Engenharia da Motocicleta. Incluem a apresentação da teoria, a construção do modelo físico e a análise dos resultados. Em geral, um exemplo de seis horas poderia ser considerado um tempo elevado para manter a atenção dos estudantes, mas, por se tratar de um fenômeno atraente e de fácil visualização, os estudantes apresentam um interesse contínuo, participando ativamente durante a formulação das hipóteses do modelo, e, posteriormente, nas discussões das consequências do movimento modelado. Ainda, ao final da disciplina, os estudantes mostram-se bastante motivados tanto para estender o modelo matemático para outras situações quanto para elaborar relatórios finais em forma de pequenas monografias.

\section{Agradecimentos}

Os autores agradecem à UFPE pela infraestrutura e à CAPES pela bolsa de mestrado.

\section{Referências}

[1] H.H. Hurt, J.V. Ouellet and D.R. Thom, Motorcycle Accident Cause Factors and Identification of Countermeasures, vol. I. (Traffic Safety Center, Los Angeles, 1981), disponível em http://bit.ly/2fw8fTA.

[2] ACEM (Association of European Motorcycle Manufacturers), Motorcycle Accidents in Depth Study (MAIDS): In-depth Investigations of Accidents Involving Powered Two-wheelers, disponível em http://www.maids-study.eu/ (2009).

[3] OMS (Organização Mundial de Saúde), Global Status Report on Road Safety 2013, disponível em http://bit.ly/1RjzbQC (2013).

[4] E.A. Vasconcellos. Risco no Trânsito, Omissão e Calamidade: Impacto do Incentivo à Motocicleta no Brasil (Edição do Autor, São Paulo, 2013), disponível em http://bit.ly/2gFavd4.

[5] CONTRAN (Conselho Nacional de Trânsito), Manual de Sinalização de Trânsito, Volume II, Sinalização Vertical de Advertência, http://bit.ly/2g9lgRo (2007).

[6] Código de Trânsito Brasileiro, Lei No 9.503, de 23 de setembro de 1997, Capítulo XV disponível em http://bit.ly/1i54cEq (1997).

[7] Honda Motocicletas do Brasil Ltda., Pilotagem Consciente: Frenagem, disponível em http://bit.ly/2fKknOd, acesso em 24/9/2016.

[8] V. Cossalter, Motorcycle Dynamics (Lulu.com, Raleigh, 2006), $\quad 2^{\text {nd }}$ ed.

[9] D.G. Wilson, Bicycling Science (MIT Press, Cambridge, 2004), $3^{\text {rd }}$ ed.

[10] G. Cocco, Motorcycle Design and Technology (Giorgio Nada Editore, Milão, 2013).

[11] M. Corno, S.M. Savaresi, M. Tanelli and L. Fabbri, Control Engineering Practice 16, 644 (2008).

[12] Y. Toyofuku, K. Matsushima, Y. Irie, H. Yonezawa and K. Mizuno, JSAE Review 15, 223 (1994).

[13] M. Baumann, T. Bächle, M. Buchholz and K. Dietmayer, IFAC-PapersOnLine 49, 291 (2016).

[14] T. Özkan, T. Lajunen, B. Doğruyol, Z. Yıldırım and A. Çoymak, Accident Analysis \& Prevention 49, 124 (2012).

[15] E.I. Vlahogianni, George Yannis and John C. Golias, Accident Analysis \& Prevention 49, 12 (2012). 